\title{
Combining Structural and Chemical Information on the Nanometer Scale by Correlative TEM and APT
}

\author{
M. Herbig, P. Choi, D. Raabe \\ Max-Planck-Institut für Eisenforschung, Department of Microstructure Physics and Alloy \\ Design, 40327 Düsseldorf, Germany
}

The strength of atom probe tomography (APT) is its capability to measure 3D chemistry with equal detection sensitivity (a few ppm) for all elements at near atomic resolution. However, its spatial resolution is material and measurement condition-dependent and is in many cases not sufficient for crystallographic identification of phases or grain orientations. Transmission electron microscopy (TEM) on the other hand, is an excellent tool for structural characterization even down to the sub $\dot{A}$ scale but an accurate quantification of the 3D local chemical composition is only possible in special cases.

For the characterization of many materials scientific phenomena both, structural and chemical information, are required on the identical sample and on the nanometer scale, viz. TEM and APT have to be performed in a correlative way. Here three examples:

1.) Displacive phase transformations, such as the transformation of austenite into martensite, lead to phases that differ in structure but not in composition. These phases can hardly be distinguished by APT. If the transformation of the metastable phase happens due to cryogenic temperatures inside the atom probe, the differentiation by APT alone is even impossible.

2.) The characterization of segregation to defects in cases where very little or no segregation occurs is a second example that can require the correlative use of TEM and APT. Undecorated dislocations, stacking faults or small twin bundles can hardly be identified by APT so that TEM is needed to prove that the defect is present within the volume analyzed by APT.

3.) The investigation of grain boundary segregation requires information on grain boundary chemistry, grain misorientation as well as grain boundary orientation.

Here we present an experimental setup that makes it possible to successively perform the two complementary techniques on the identical sample. Compared to former approaches [1, 2], mainly the practicability and quality of TEM characterization is improved. The core of the setup is a modified high angle single-tilt TEM retainer that holds a TEM grid on which a batch of atom probe tips is placed and shaped by standard focus ion beam (FIB) lift-out techniques. The use of an adapter during FIB milling makes it possible to shape the atom probe tips in such a way that their axes of rotational symmetry are parallel to the TEM retainer's tilt axis, providing constant focus condition during TEM single-tilt operation. Two examples for the STEM imaging quality attained by this approach are given in figure 1. The setup was designed for minimum risk during grid manipulation, making it possible to insert the samples into a double-tilt TEM holder and thereby enabling aligned diffraction and high resolution TEM experiments if needed.

The power and versatility of this correlative TEM/APT approach will be demonstrated with an example for each of the above mentioned cases:

1.) The identification of austenitic and martensitic grains for the investigation of carbon partitioning phenomena during a quenching and partitioning heat treatment. 
2.) The characterization of segregation to twin bundles in twinning induced plasticity steels.

3.) And the characterization of the dependence of the carbon grain boundary excess in ferrite on grain misorientation and grain boundary normal for a data set of more than 100 grain boundaries.

The authors acknowledge financial support by the German Research Foundation in the framework of the SFB 761 "steel ab initio" and their collaborators Y. Toji, R.K.W. Marceau, I. Gutierrez-Urrutia, Y. Li and S. Goto.

[1] B. W. Krakauer, J. G. Hu, S.-M. Kuo, R. L Mallick, A. Seki, D. N. Seidman, J. P. Baker and R. J. Loyd, Rev. Sci. Instrum. 61:11 (1990), p. 3390.

[2] P. J. Felfer, T. Alam, S. P. Ringer and J. M. Cairney, Microscopy Research and Technique 75 (2012), p. 484.

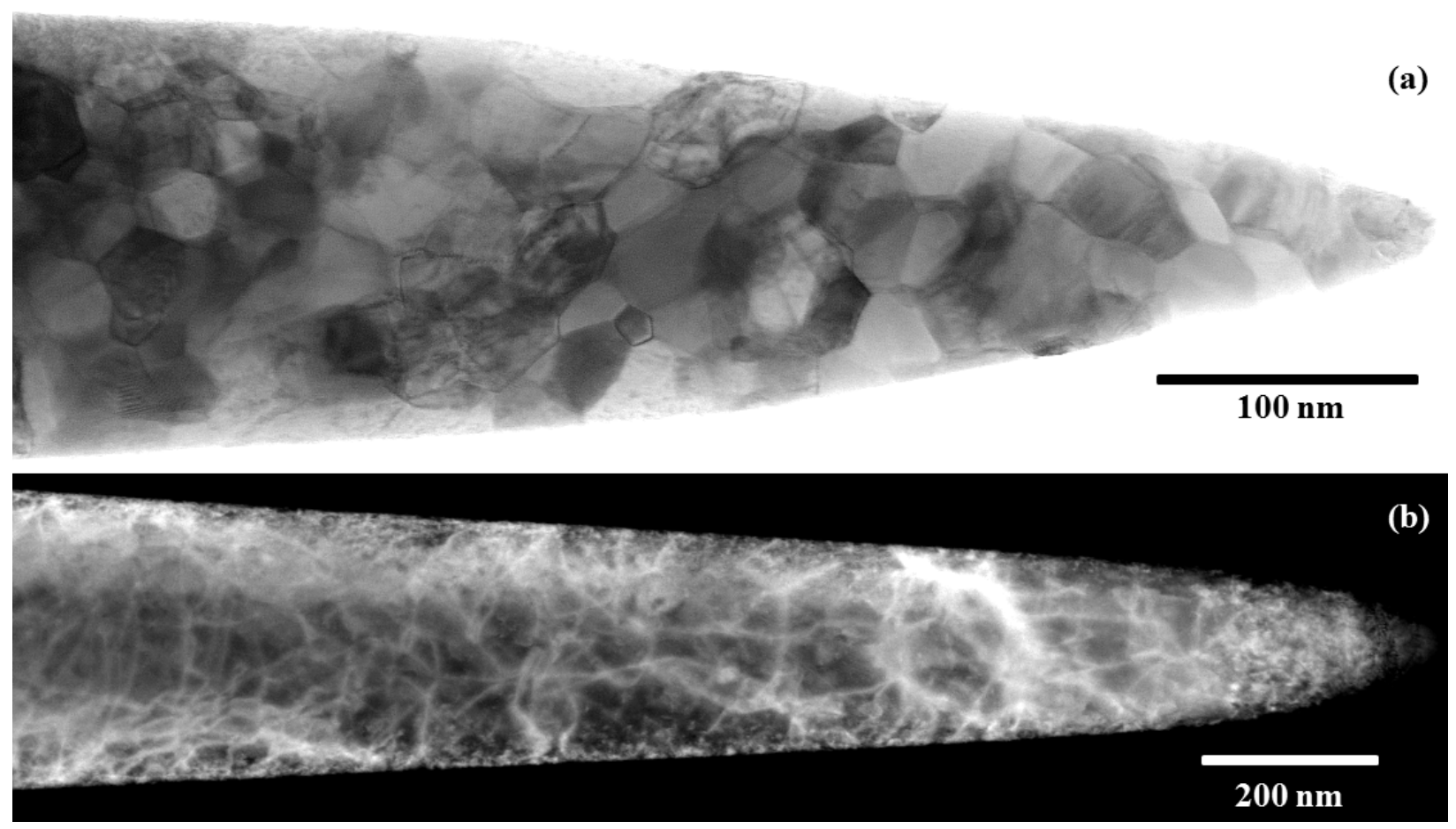

Figure 1: STEM micrographs of atom probe tips. (a) Bright-field image of columnar grains in high strength steel with grain sizes of 20-40 nm perpendicular to the beam direction. (b) Darkfield image of dislocation network in martensitic steel. 